\title{
THREE-DIMENSIONAL DIRECT NUMERICAL SIMULATION (DNS) OF TAYLOR BUBBLES RISING IN NON-NEWTONIAN ENVIRONMENTS
}

\author{
Ahmad Amani $^{1}$, Jesús Castro ${ }^{1}$ AND Assensi Oliva ${ }^{1}$ \\ ${ }^{1}$ Heat and Mass Transfer Technological Center (CTTC), \\ Universitat Politécnica de Catalunya-Barcelona Tech (UPC), \\ ESEIAAT, Colom 11, 08222 Terrassa (Barcelona), Spain. ahmad.amani@upc.edu
}

Key words: Direct Numerical Simulation (DNS), Taylor bubbles, non-Newtonian Environment

\begin{abstract}
Three-dimensional numerical simulation of Taylor gas bubbles as primary unites of slug flow patterns rising in non-Newtonian environments is performed in the context of Direct Numerical Simulation (DNS) of the governing equations, where the whole physics of fluid motions will be taken into account. State-of-the-art numerical tools are proposed to tackle the numerical challenges in the DNS study of this problem. E.g. a coupled level-set volume-of-fluid (CLSVOF) interface capturing method is used to solve the topological changes of the interface. Physical formulations are integrated with moving-mesh (MM) technique to decrease the computational cost of 3D simulations and adaptivemesh-refinement (AMR) technique to increase the local accuracy around the interface. The governing equations are solved using High-Performance Computing (HPC) parallel approaches. To the best of the authors' knowledge, this is the first work dealing with three-dimensional direct numerical simulation of Taylor bubbles rising in non-Newtonian environments.
\end{abstract}

\section{INTRODUCTION}

Slug flow is a frequently found multiphase flow pattern when a system of gas bubble suspended in a matrix fluid concurrently flow in a pipe. For example in side-stream (airlift) membrane bioreactors (MBR), the vertical tubular membranes are located outside of the bioreactor, the sludge is pumped from the bioreactor to the membrane modules, and the air is added at the base of the membranes to gain a two-phase slug flow of appropriate regime. Taylor bubbles are the primary units of this pattern. Taylor bubbles are characterised by their width which almost fills the cross-section of the tube and by their length which is usually above 2 or 3 tube diameters long. They are separated from the tube wall by a thin matrix film which expands when it reaches the rear end of the bubble. This expansion leads to the formation of a wake.

Starting by the original works of Davies and Taylor [1] and White and Beardmore [2], various methods have been used to solve the problem of Taylor bubbles rising in a Newtonian matrix, including experimental research [3, 4, 5, 6], theoretical models [7, 8], in addition to numerical approaches of Volume of Fluid (VOF) [9], Front Tracking [10], Lattice Bolzmann (LBM) [11, 12], conservative level-set [13] and others methods $[3,14]$. The dimensionless group of governing parameters could define the hydrodynamics of a Taylor bubble in a Newtonian matrix as below: 
- Ratio of gravitational forces and the surface tension embedded in Eötvös number as:

$$
E o=g\left(\rho_{m}-\rho_{b}\right) D^{2} / \sigma
$$

- Ratio of inertial and gravitational forces embedded in Froude number as:

$$
F r=U_{T B} / \sqrt{g D\left(\rho_{m}-\rho_{b}\right) / \rho_{m}}
$$

- Property group combining gravitational, viscous and surface tension forces embedded in Morton number as:

$$
M=g \mu_{m}^{4}\left(\rho_{m}-\rho_{b}\right) / \rho_{m}^{2} \sigma^{3}
$$

where parameters $\rho, \mu, g, \sigma, U_{T B}$ and $D$ are density, dynamic viscosity, gravitational acceleration, surface tension coefficient, terminal velocity of the bubble and the column diameter, respectively. In these formulations, the index $m$ stands for matrix and index $b$ stands for bubble. In case of a non-Newtonian matrix, the spatial variation of the fluid viscosity and as a result the spatial variation of Morton number or incorporation of elastic behaviour in the matrix appends to the complexity of the problem while granting additional controlling parameters for the rising of the Taylor bubble.

Compared to the related topic in Newtonian fluids, state of the art about the characteristics of Taylor bubbles rising in non-Newtonian matrices is still in an early stage. Despite vast application of Taylor bubbles rising in non-Newtonian in wells, processing of pol tion, air-lift reactors, rever Therefore, there is a need The research area of Taylor-bubbtes rising in non-1

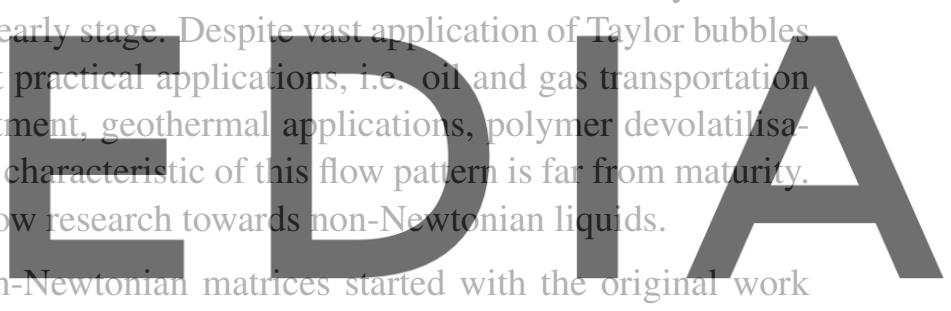
of Rosehart, Rhodes, and Scott [15], where they measured the void fraction, velocity and frequency of

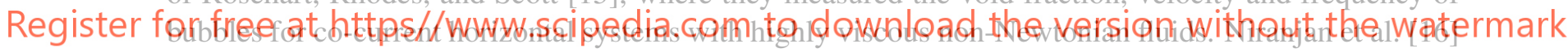
investigated mass transfer of gas from vertically rising Taylor bubbles in Newtonian and non-Newtonian environments. Sousa et al. [17] used a particle image velocimetry (PIV) and shadowgraphy technique to study the flow in negative wake of a Taylor bubble in non-Newtonian aqueous solutions of 0.8 and $1.0 \%$ carboxymethylcellulose (CMC). They have witnessed the negative wake formed downstream of the bubble and cusp-shape rear of the bubble, a phenomena that was observed before for rising of the bubble in viscoelastic matrices. In another work, Sousa [18] investigated the flow around an individual Taylor bubble rising in CMC solutions with weight percentage varying from 0.1 to $1.0 \mathrm{wt} \%$. They have extracted the flow patterns and velocity profiles in different sections of the problem. They have performed similar study for a Taylor bubble rising in stagnant polyacrylamide (PAA) solutions [19].

Despite frequent use in different applications, the area of Taylor bubbles rising in non-Newtonian matrices is far from maturity. To the best of the authors knowledge, there has not been any comprehensive three-dimensional numerical study in this field. The rest of the paper is organized as follow: mathematical formulation and numerical methods are presented in section 2, numerical experiments are discussed in section 3 and conclusion remarks in section 4. 


\section{Mathematical formulation and numerical method}

Navier-Stokes equations are used to describe the conservation of mass and momentum of two incompressible immiscible Generalized-Newtonian-Fluid (GNF) on a spacial domain $\Omega$ with boundary $\partial \Omega$ on a moving domain as following [20,21, 22, 23]:

$$
\begin{aligned}
\frac{\partial}{\partial t}(\rho \mathbf{v})+\nabla \cdot\left(\rho \mathbf{v}\left(\mathbf{v}-\mathbf{v}_{d}\right)\right) & =-\nabla p+\nabla \cdot\left(\mu_{s}(\dot{\gamma})\left(\nabla \mathbf{v}+(\nabla \mathbf{v})^{T}\right)\right)+\rho \mathbf{g}+\sigma \kappa \mathbf{n} \delta_{\Gamma} \\
\nabla \cdot \mathbf{v} & =0
\end{aligned}
$$

where $\mathbf{v}$ is the velocity field, $\mathbf{v}_{d}$ the velocity of the domain, $p$ pressure field, $\delta_{\Gamma}$ the Dirac delta function concentrated at the interface $(\Gamma), \mathbf{n}$ the unit normal vector outward to interface, and $\kappa$ the interface curvature. A moving mesh approach is used to minimize the size of the computational domain in the gravitational direction, thus constantly moving the domain, so its center coincides with the mass center of the bubble [24]. Careful implementation of the boundary condition in the mesh moving direction has taken into account to conserve the mass of the fluids during the moving process. In this formulation, $\mu_{s}(\dot{\gamma})$ is the apparent viscosity of the fluids described in the context of generalized Newtonian fluid (GNF) models, with $\dot{\gamma}$ as shear-rate tensor of the fluid defined by:

$$
\dot{\gamma}=\nabla \mathbf{v}+(\nabla \mathbf{v})^{T}
$$

In this study, Carreau-Yasuda GNF model is used to describe the relation of apparent viscosity $\mu_{s}$, with the shear-rate tensor $\dot{\gamma}$ as
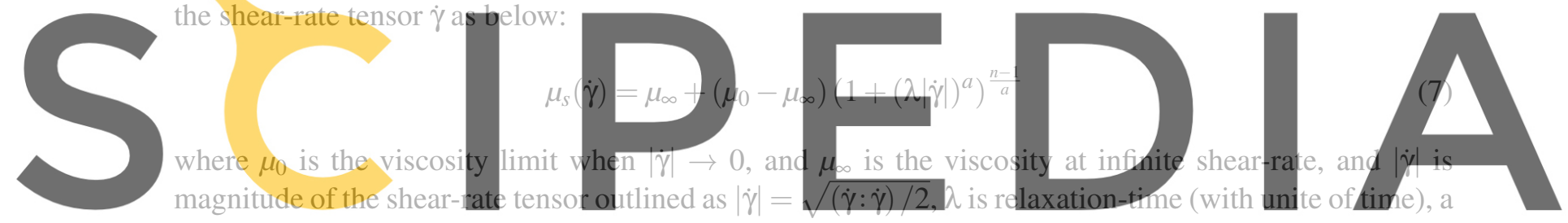

and $\mathrm{n}$ are non-dimensional power-index coefficients. Taking into account that mass, density and viscosity Register far fpeed

$$
\rho=\rho_{1} H+\rho_{2}(1-H) \quad \mu_{s}=\mu_{s 1} H+\mu_{s 2}(1-H)
$$

where $H$ is the Heaviside step function taking the value one in dispersed phase and zero elsewhere [25, 26]. In this paper, a geometrical volume-of-fluid approach in the context of a finite-volume method for unstructured grids is used, where the interface is captured implicitly using a color function scalar field $C(\mathbf{x}, t)$, representing the volume fraction of a phase inside each cell of the discretized domain at a given time:

$$
C(\mathbf{x}, t)= \begin{cases}1 & \text { cell filled with phase } 1 \\ 0 & \text { cell filled with phase } 2\end{cases}
$$

In this formulations, cells with $0<C(\mathbf{x}, t)<1$ are known as interface cells. For immiscible fluids where their movement is defined by the velocity field extracted from Navier-Stokes equations, the colorfunction motion is solved by an advection equation as:

$$
\frac{\partial C}{\partial t}+\nabla \cdot\left(C\left(\mathbf{v}-\mathbf{v}_{d}\right)\right)=0
$$


There are two methods widely used for solution of this equation, (i) using standard numerical convection schemes, known as Algebraic VOF methods (ii) using geometrically reconstructed interface calculated based on volume fraction values at interface cells, known as Geometric VOF methods. In this work, a geometric VOF approach is used to solve the advection equation of color-function by first reconstructing the interface at the each grid cell and then using this interface to calculate the total volumetric flux of phases across the faces of each interface cell. In this work, a point-cloud approach is being used to calculate the normal vectors of the interface cells which are used in a piecewise linear interface calculation (PLIC) estimate of the interface. The resulting flux polyhedrons are constructed using the Lagrangian trajectories of the cell-vertex velocities Jofre et al. [27]. In the classical formulation, the curvature is calculated as divergence of the gradient of color-function: $\kappa=\nabla \cdot(\nabla C /|\nabla C|)$. However, given the fact that the colorfunction in VOF is discontinues by definition, applying derivative type operations to this discontinuous function leads to numerical errors. To circumvent this issue, a sign distance function $(\phi)$ representing the minimum distance of each cell's center from the PLIC interface is evaluated in a neighbouring area of the interface. The curvature is then calculated using this continuous auxiliary distance-function as: $\kappa=\nabla \cdot(\nabla \phi /|\nabla \phi|)$.

Finite-volume (FV) approach is used to discretize the Navier-Stokes and VOF-advection equations on a collocated grid, so all the computed variables are stored at centroids of the cells. A central difference (CD) scheme is used to discretize the diffusive and convective fluxes at the faces. A distanceweighted linear interpolation is used to calculate the face values of physical properties. The gradients are computed at the cell centroids using the least-squares method. At discretized level, physical properties are regularized in the context of the VOF method. Therefore a linear average is used for density as $\rho=\rho_{1} C+\rho_{2}(1-C)$, and a harmonic average is used for viscosity average of viscosity has linear average [20]. A classical fractional step proj solve the velocity-pressure coupling.

tive, and gravitational terms and also by explicit treatment of the surface tension as used by [22] with $\alpha$

Register for free at https//www.scipedia.com to download the version without the watermark

$$
\Delta t \equiv \alpha \times \min \left(\frac{h}{\left\|V_{P}\right\|}, \sqrt{\frac{h}{|\mathbf{g}|}}, h^{3 / 2} \sqrt{\frac{\rho_{1}+\rho_{2}}{4 \pi \sigma}}\right)
$$

The velocity and pressure fields are calculated using a fractional-step method. A second-order implicit Crank-Nicolson scheme is used to discretize the diffusion term of equation ?? while a second-order Adams-Bashforth scheme is used on convective, gravity and surface tension terms. In order to increase the calculation's accuracy, an Adaptive Mesh Refinement (AMR) approach is used to locally refine the mesh in the areas close to the interface with a distance of $3 \mathrm{~h}$ with the interface cells, where $\mathrm{h}$ stands for characteristic size of the cell. The numerical methods are implemented in an in-house parallel c++/MPI code called TermoFluids [29]. Validations and verifications of the numerical methods used in this work have been reported in $[27,20,22,23,21,25,24]$. 


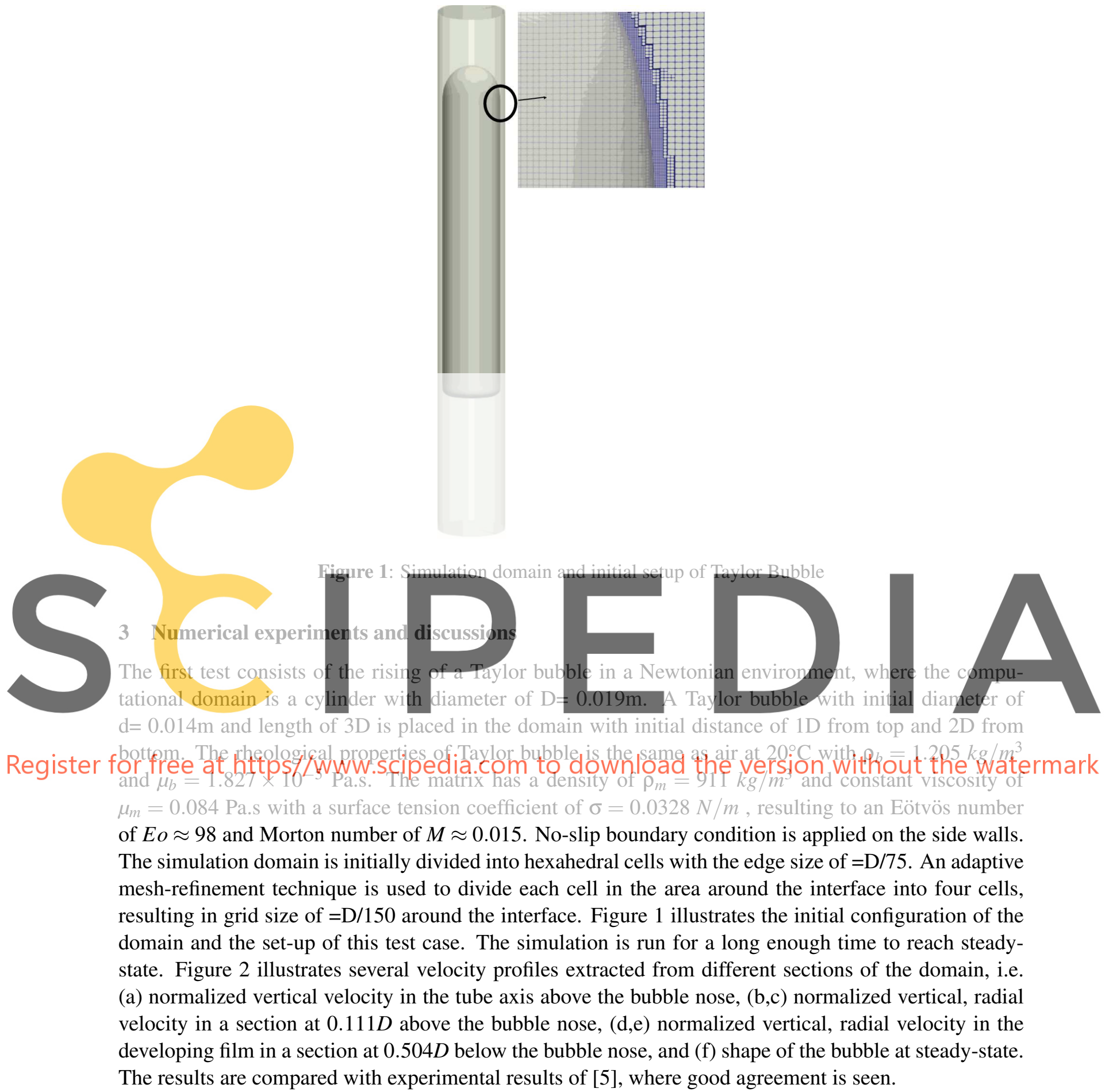

Next, in a similar setup, simulation of Taylor bubble rising in non-Newtonian environments is performed. A cylindrical domain with diameter of $\mathrm{D}=0.032 \mathrm{~m}$ is used and discretized into hexahedral cells with the same size of $\mathrm{h}=\mathrm{D} / 75$. An air Taylor bubble with initial diameter of $\mathrm{d}=0.027328 \mathrm{~m}$ and length of 


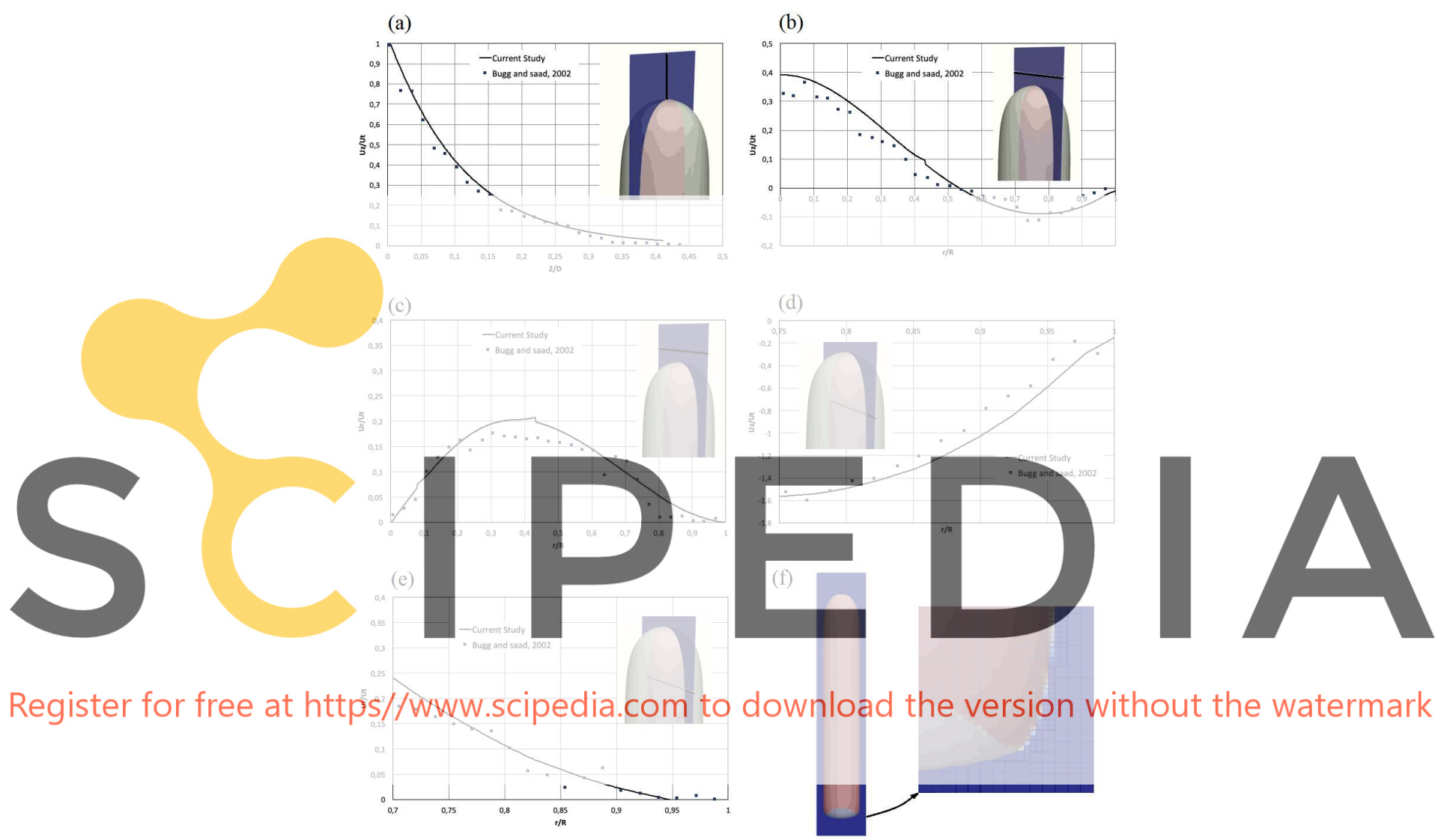

Figure 2: (a) Normalized vertical velocity in the tube axis above the bubble nose, (b,c) Normalized vertical, and radial velocity in a section at $0.111 D$ above the bubble nose, $(\mathrm{d}, \mathrm{e})$ Normalized vertical, radial velocity in the developing film in a section at $0.504 D$ below the bubble nose, (f) shape of the bubble at steady-state. 


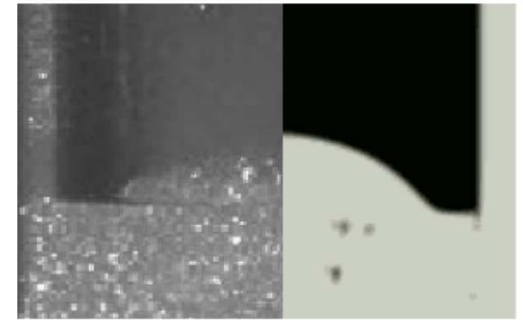

(a)

(b)

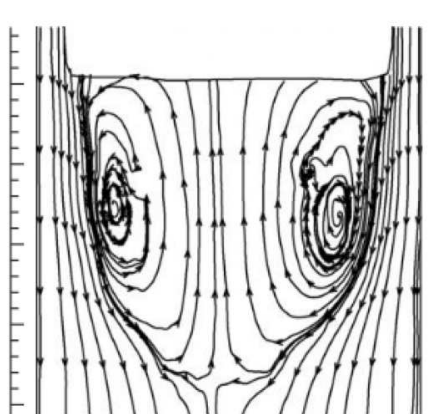

(c)

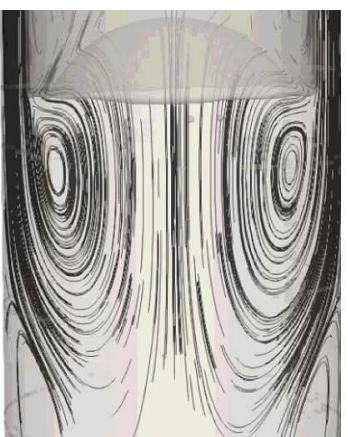

(d)

Figure 3: Final shape of the tail of the Taylor bubble along with the vortical structures in the matrix environment in $(a, c)$ experimental results of [30], and $(b, d)$ simulations of current study.

3D is placed inside the domain with initial distance of 1D from top and 2D from bottom. A shearthinning Carboxymethylcellulose (CMC) $0.4 \mathrm{wt} \%$ polymer solution used as matrix fluid with density of $\rho_{m}=996.9 \mathrm{~kg} / \mathrm{m}^{3}$ and surface tension coefficient of $\sigma=0.0328 \mathrm{~N} / \mathrm{m}$. The viscosity of the matrix follows the Carreau-Yasuda model of equation 7 with $\left(\mu_{\infty}, \mu_{0}, \lambda, a, n\right)=\left(10^{-3}, 0.1102,0.1099,0.8087,0.6751\right)$, as presented in Mulhem, Schulte, and Fritsching [30], thus:

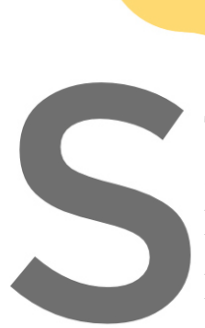

$$
\mu_{s}(\dot{\gamma})=10^{-3}+0.109\left(1+(0.1099|\dot{\gamma}|)^{0.8087}\right)^{-0.4}
$$

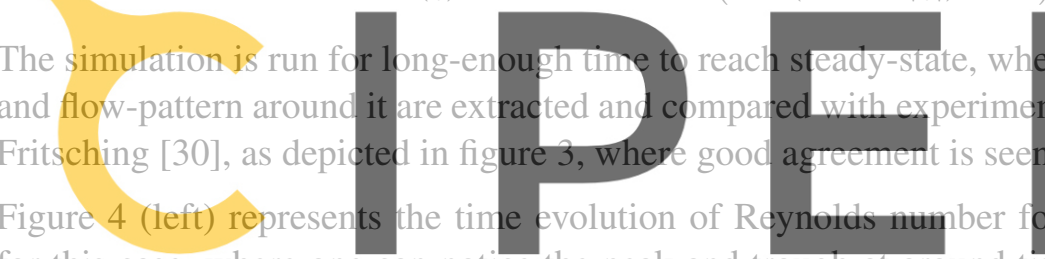

for this case, where one can notice th

to steady-state where a $R e_{\text {steady }} \approx 18$ is seen. Figure 4 (right) illustrates the vertical velocity contours

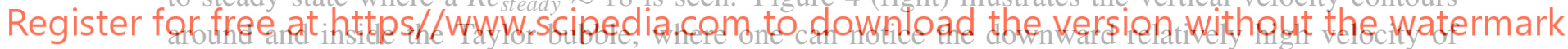

the film in the area between the Taylor bubble and the wall. Figure 5 illustrates the velocity magnitude

contours at control-plane P1, 0.15275D above the nose of Taylor bubble as well as vorticity magnitude contours in two planes of P2 and P3 located at $0.1725 \mathrm{D}$ and $0.5250 \mathrm{D}$ below the tail of Taylor bubble. As can be seen, the velocity magnitude above the nose is almost unaffected by the movement of Taylor bubble, thus the location of the upper-wall at 1D distance from the nose of Taylor bubble has a negligible effect on the evolution of the Taylor bubble. The contours at cross-sections below the Taylor bubble also shows a rapid reduction in the vorticity magnitude as we go further away from the tail, thus the location of the bottom wall in a distance of 2D from the Taylor bubble has also negligible effect on the evolution of the interface.

\section{Conclusions}

In this paper, a coupled level-set volume-of-fluid interface capturing approach combined with movingmesh and adaptive-mesh-refinement methods was developed and used in a finite-volume framework to simulate challenging problem of Taylor bubble rising in Newtonian/non-Newtonian environments. Implementing moving-mesh approach has immensely reduced the computational cost of the simulations 

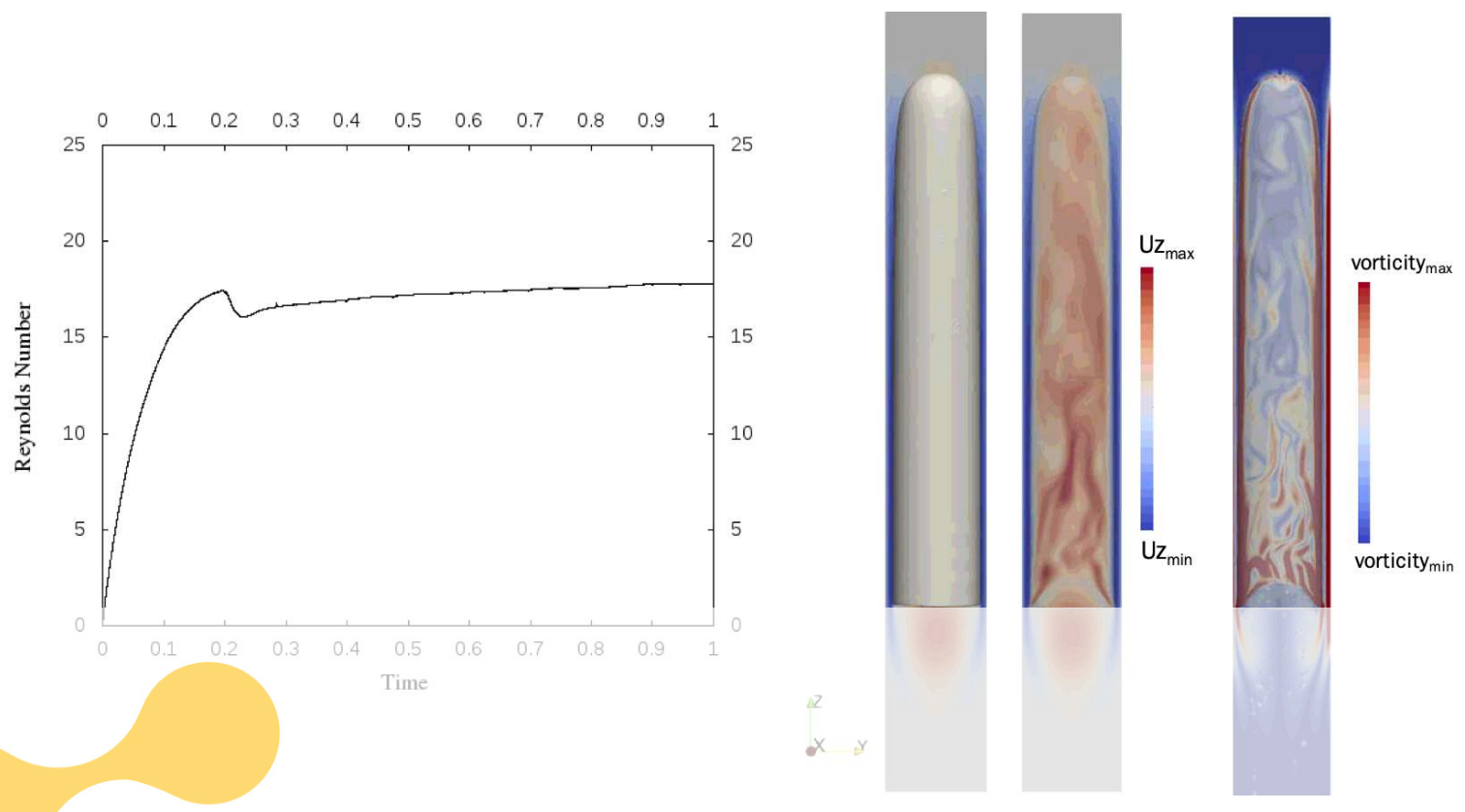

Figure 4: Left: time evolution of the Reynolds number, and right: vertical velocity and vorticity magnitude contours around and inside the Taylor bubble for the case of rising Taylor bubble in CMC 0.4wt\% polymeric solution.
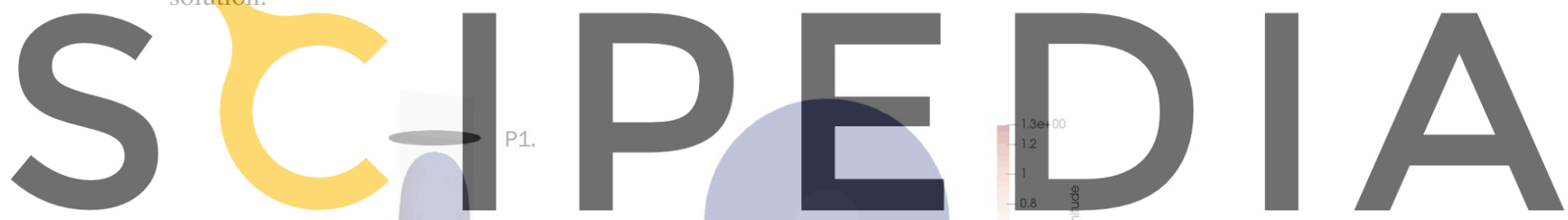

Register for free at https//www.scipedia.com to download the version without the watermark
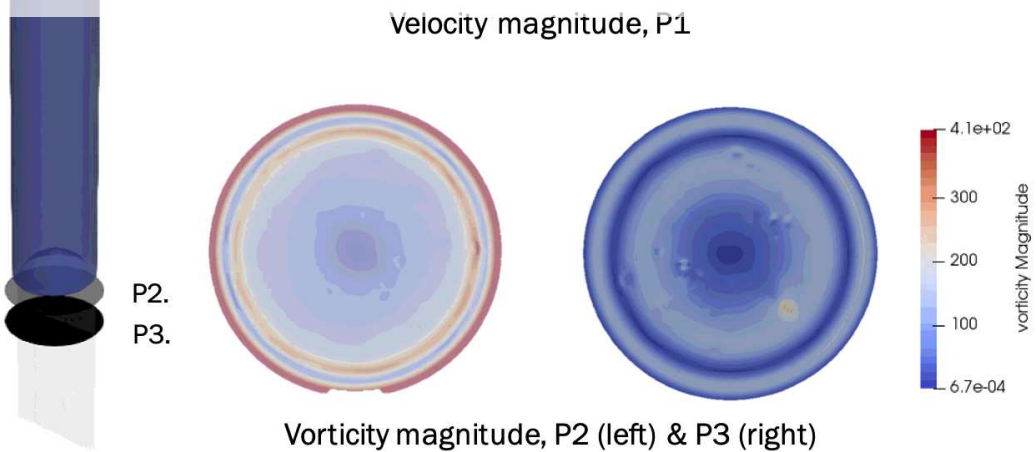

Figure 5: Velocity magnitude contour at control-plane P1, 0.15275D above the nose of Taylor bubble, along with vorticity magnitude contours in two planes of P2 and P3 located at $0.1725 \mathrm{D}$ and $0.5250 \mathrm{D}$ below the tail of Taylor bubble. 
by reducing the required domain height, while adaptive-mesh-refinement has improved the accuracy of the topological change of interface to a greater scale. Coupled level-set volume-of-fluid improves the evaluation of geometrical properties of the interface. The numerical techniques were validated against experimental results available in the literature were good agreement was seen, while the effect of viscoelastic environments on rising of Taylor bubbles is left for future works.

\section{Acknowledgments}

The work has been financially supported by a competitive R+D project (ENE2017-88697-R) by the Spanish Research Agency.

\section{References}

[1] R. M. Davies and G. Taylor. "The mechanics of large bubbles rising through liquids in tubes". In: Proc. R. Soc. (1950), pp. 375-390.

[2] E. T. White and R. H. Beardmore. "The velocity of rise of single cylindrical air bubbles through liquids contained in vertical tubes". In: Chemical Engineering Science 17.5 (1962), pp. 351-361.

[3] R. Abiev. "Bubbles velocity, Taylor circulation rate and mass transfer model for slug flow in milli- and microchannels". In: Chemical Engineering Journal 227 (2013), pp. 66-79.

[4] S. Bhusan et al. "Rise of Taylor bubbles through narrow rectangular channels". In: Chemical Engineering Journal 155.1 (2009), pp. 326-332.

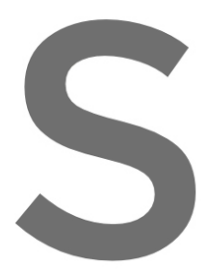

[5] J. D. Bugg and G. A. Sald. "The velocity field around a yiscous fluid: Numerical and experimental results". In: phase Flow (2002)

[6] C. E. Shosho and M. E. Ryan. "An experimental study inclined tubes"

[7] Y. B. Zudin. "Analytical solution of the problem of the rise of a Taylor bubble". In:

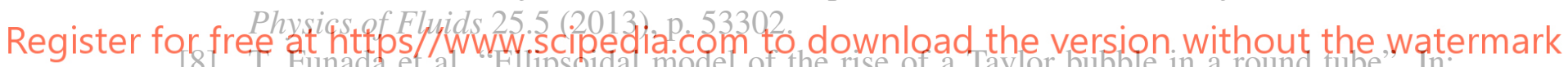
International Journal of Multiphase Flow 31.4 (2005), pp. 473-491.

[9] M. Dang et al. "Formation characteristics of Taylor bubbles in a microchannel with a converging shape mixing junction". In: Chemical Engineering Journal 223 (2013), pp. 99109.

[10] S. Quan. "Co-current flow effects on a rising Taylor bubble". In: International Journal of Multiphase Flow 37.8 (2011), pp. 888-897.

[11] A. Kuzmin et al. "Lattice Boltzmann study of mass transfer for two-dimensional Bretherton/Taylor bubble train flow". In: Chemical Engineering Journal 225 (2013), pp. 580596.

[12] L. Amaya-Bower and T. Lee. "Numerical simulation of single bubble rising in vertical and inclined square channel using lattice Boltzmann method". In: Chemical Engineering Science 66.5 (2011), pp. 935-952. 
[13] E. Gutiérrez et al. "Numerical study of Taylor bubbles rising in a stagnant liquid using a level-set/moving-mesh method”. In: Chemical Engineering Science 164 (2017), pp. 158177.

[14] N. V. Ndinisa, D. E. Wiley, and D. F. Fletcher. "Computational fluid dynamics simulations of Taylor bubbles in tubular membranes model validation and application to laminar flow systems". In: Chemical Engineering Research and Design 83.1 A (2005), pp. 40-49.

[15] R. G. Rosehart, E. Rhodes, and D. S. Scott. "Studies of gas $\rightarrow$ liquid (non-Newtonian) slug flow: void fraction meter, void fraction and slug characteristics". In: The Chemical Engineering Journal 10.1 (1975), pp. 57-64.

[16] K. Niranjan et al. "Liquid-phase controlled mass transfer from a gas slug". In: Chemical Engineering Science 43.6 (1988), pp. 1247-1252.

[17] R. G. Sousa et al. "Flow in the negative wake of a Taylor bubble rising in viscoelastic carboxymethylcellulose solutions: Particle image velocimetry measurements". In: Journal of Fluid Mechanics 511 (2004), pp. 217-236.

[18] R. Sousa. "Flow of Taylor Bubbles Rising in Stagnant Non-Newtonian Fluids". In: (2005), p. 391.

[19] R. G. Sousa et al. "Flow around individual Taylor bubbles rising in stagnant polyacrylamide (PAA) solutions". In: Journal of Non-Newtonian Fluid Mechanics 135.1 (2006), pp. 16-31.

[20] A. Amani et al. "Numerical study of droplet deformation in shear flow using a conserva-

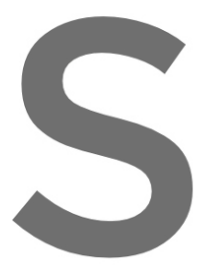
tive level-set meth

[21] E. Gutiérrez et complex geometrie Chemical Enginee

[22] A. Amani et al regimes". In: Chemical
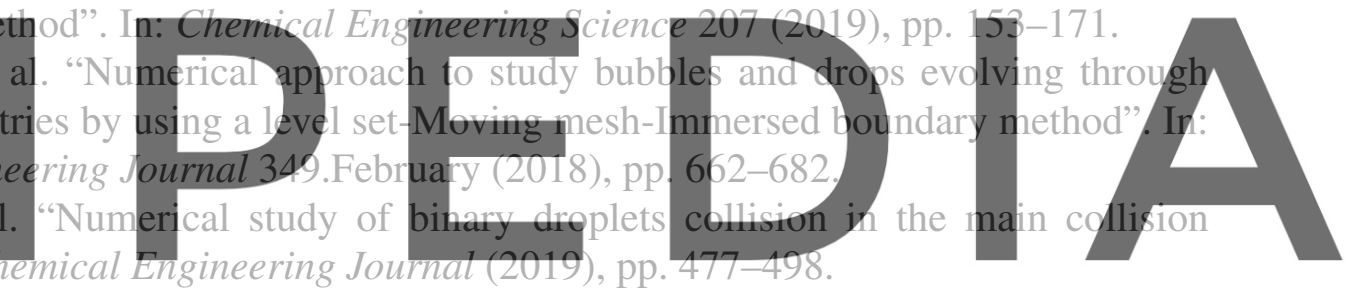

[23] A. Amani et al. "A numerical approach for non-Newtonian two-phase flows using a

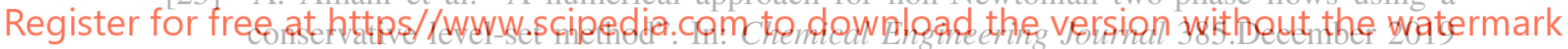
(2020), p. 123896.

[24] A. Amani et al. "DNS of un-equal size dropiets coliision using a moving-mesh/levelset method". In: ERCOFTAC workshop direct and large eddy simulation 12 (DLES 12). Madrid, Spain, 2019.

[25] A. Amani et al. "A Study on Binary Collision of GNF Droplets Using a Conservative Level-Set Method". In: 6th European Conference on Computational Mechanics (ECCM 6)- 7th European Conference on Computational Fluid Dynamics (ECFD 7). Glasgow, UK, 2018.

[26] A. Amani. "Numerical simulation of Newtonian/non-Newtonian multiphase flows : deformation and collision of droplets". PhD thesis. Universitat Politècnica de Catalunya, 2019.

[27] L. Jofre et al. "A 3-D Volume-of-Fluid advection method based on cell-vertex velocities for unstructured meshes”. In: Computers and Fluids 94 (2014), pp. 14-29. 
[28] A. J. Chorin. "Numerical solution of the Navier-Stokes equations". In: Mathematics of Computation 22.104 (1968), pp. 745-745.

[29] Http://www.termofluids.com/. Termo Fluids S.L.

[30] B. Mulhem, G. Schulte, and U. Fritsching. "Solid-liquid separation in suspension atomization”. In: Chemical Engineering Science 61.8 (2006), pp. 2582-2589. 